\title{
CBDB: The codon bias database
}

\author{
Adam Hilterbrand ${ }^{1}$, Joseph Saelens ${ }^{2}$ and Catherine Putonti ${ }^{1,2,3^{*}}$
}

\begin{abstract}
Background: In many genomes, a clear preference in the usage of particular codons exists. The mechanisms that induce codon biases remain an open question; studies have attributed codon usage to translational selection, mutational bias and drift. Furthermore, correlations between codon usage within host genomes and their viral pathogens have been observed for a myriad of host-virus systems. As such, numerous studies have investigated codon usage and codon bias in an effort to better understand how species evolve. Numerous metrics have been developed to identify biases in codon usage. In addition, a few data repositories of codon bias data are available, differing in the metrics reported as well as the number and taxonomy of strains examined.
\end{abstract}

Description: We have created a new web resource called the Codon Bias Database (CBDB) which provides information regarding the codon bias within the set of highly expressed genes for 300+ bacterial genomes. CBDB was developed to provide a resource for researchers investigating codon bias in bacteria, facilitating comparisons between strains and species. Furthermore, the site was created to serve those studying adaptation in phage; the genera selected for this first release of CBDB all have sequenced, annotated bacteriophages. The annotations and sequences for the highly expressed gene set are available for each strain in addition to the strain's codon bias measurements.

Conclusions: Comparing species and strains provides a comprehensive look at how codon usage has been shaped over evolutionary time and can elucidate the putative mechanisms behind it. The Codon Bias Database provides a centralized repository of look-up tables and codon usage bias measures for a wide variety of genera, species and strains. Through our analysis of the variation in codon usage within the strains presently available, we find that most members of a genus have a codon composition most similar to other members of its genus, although not necessarily other members of its species.

\section{Background}

\section{Why does codon bias exist?}

The redundancy within the genetic code accommodates a variable number of codons to encode for the same amino acid. Codon usage biases have been found to exist, ranging from relatively neutral to extremely strong [1-6]. Debate within the scientific community continues as to exactly why codon biases exist. Theories based on translational selection, mutational biases, and drift have all been found to contribute to codon biases [7-11]. Sequence-based analysis revealed that within organisms having a biased genome, the most frequently occurring codons often reflect the most abundant transfer RNA (tRNA) available [12-15].

\footnotetext{
* Correspondence: cputonti@luc.edu

'Department of Biology, Loyola University Chicago, 1032 W Sheridan Road, Chicago IL 60660, USA

2Bioinformatics Program, Loyola University Chicago, 1032 W Sheridan Road, Chicago IL 60660, USA

Full list of author information is available at the end of the article
}

Furthermore, through direct molecular manipulation protein-throughput was increased by designing coding sequences to utilize the most abundant tRNAs [16-18]. These bioinformatic and experimental studies suggest that translational selection may be the primary factor shaping codon usage.

Correspondences between codon usage and tRNA availability may signify selection for translational accuracy, selection for translational efficiency, or both [19-30]. Theories for selection for translational accuracy assume that the codon with the highest tRNA abundance has a lower missense error rate than its synonymous codons, considering tRNA gene copy numbers of both cognate and near-cognate tRNA abundances (e.g. [11,31]). If codon bias exists as a result of selection to maximize protein-throughput, codon usage often reflects available tRNAs. Because codon bias is often strongest within highly expressed genes and there exists a correspondence
C Biomed Central

(C) 2012 Hilterbrand et al.; licensee BioMed Central Ltd. This is an Open Access article distributed under the terms of the Creative Commons Attribution License (http://creativecommons.org/licenses/by/2.0), which permits unrestricted use distribution, and reproduction in any medium, provided the original work is properly cited. 
between generation time and the strength of codon bias within these highly expressed genes [6], it is believed that selection for translational efficiency plays some role in determining a species' codon usage. Moreover, recent studies have shown that the usage of particular synonymous codons can also impact protein folding or misfolding [32-34].

As the abundances of individual tRNAs vary from one species to the next, so too do the preferences in codons. Translational selection seems most prevalent in viral species. Correspondence in virus-host codon usage has been observed in DNA-based, RNA-based, and retro-transcribing viruses (e.g. [35-39]). This observation is not isolated to eukaryote-infecting viruses; bacteriophages also exhibit codon biases similar to their host species (e. g. [40-42]). Given that viruses are heavily dependent upon their host for biosynthesis, utilizing the most prevalent tRNAs within the host would likely give the virus a translational advantage.

\section{Examining codon bias}

In order to quantify the bias present within a species, a number of metrics have been developed. Calculating the codon usage frequencies within the genome can reveal biases, in particular when comparing these usage profiles to expected usage patterns and/or the usage profiles of synonymous codons, e.g. the frequency of preferred codons (FOP) metric [13], the synonymous codon usage order (SCUO) metric [43], and the \%MinMax algorithm [44]. In order to compare the strength of biases within and between species, the relative synonymous codon usage (RSCU) and the geometric mean of the RSCU values, the codon adaptation index (CAI), were developed in which codon usage frequencies within highly expressed genes (HEGs) are specifically examined $[6,23,45]$. Numerous extensions of the CAI metric have been proposed, e.g. the self-consistent codon index (SCCI) [46] and relative codon adaption index (rCAI) [47]. Rather than looking at the codons themselves to ascertain biases, a second approach exists in which biases are assessed relative to individual tRNA abundances. The tRNA adaptation index (tAI), also inspired by the CAI metric, considers not only the gene copy number of the tRNA with the perfectly matched anticodon but also those tRNAs which can bind imperfectly [24]. The "local tAI" metric takes a similar approach, however the tAI measure is averaged for sliding windows across the gene rather than for the whole gene sequence [26]. In many species the codon usage in HEGs matches tRNA abundance $[12,48]$.

Given the information encoded within the usage of codons, a number of tools and databases have been developed for analyzing the codon and tRNA content of genic and genomic sequences. CAIcal [49], CodonExplorer [50], and CodonW [51] calculate CAI values for user input sequences while E-CAI [52] calculates the expected CAI values by generating random sequences with a $\mathrm{G}+\mathrm{C}$ content and amino acid composition similar to the user input sequence. The application CodonO [53] can analyze individual genomes or compare genomes using the SCUO metric. JCat [54] and GCUA [55] both calculate the RSCU values of user input sequences relative to a reference organism's usage profile. The RSCU values for many species are contained in the Codon Usage Database [56]; this collection, however, does not appear to have been updated since June 15, 2007. The Microbial Genome Codon Usage Database [57] and Prokaryotes Codon Usage Database [58] have lookup tables of codon counts and frequencies for over 500 and 800 species, respectively (as of June 2011). The most comprehensive database of codon statistics for individual microbial strains can be found in the Codon Usage Bias Database (CUB-DB) [59]; individual links guide the user to individual strain values for many of the aforementioned metrics as well as two metrics developed by the database's author [60].

\section{The codon bias database}

We have developed a new web resource called the Codon Bias Database (CBDB) which lists RSCU, normalized RSCU, and frequency biases (FB) values for $300+$ and counting bacterial strains. The genera selected for this first release of CBDB all have sequenced, annotated bacteriophages, thus providing a reference for those studying adaptation in phage. Following the metric proposed by Paul Sharp and collaborators $[6,23,45]$, analysis is performed for the set of highly expressed genes (HEGs) and these gene sets have been manually curated. CBDB is organized in such a way to easily accommodate codon usage comparisons between strains and species.

\section{Construction and content}

In this initial release, over 300 strains belonging to 17 genera were selected. The FASTA format (".fna) and protein coding gene (".ptt) files were downloaded from the NCBI FTP site [60]. Code developed in $\mathrm{C}++$ generated *. ptt files containing only the annotations of the HEGs which includes 40 genes previously used for analysis of codon bias [6]; these genes encode for the translation elongation factor Tu (tufA), Ts (tsf) and G (fusA) as well as 37 ribosomal proteins ( $r p l A-r p l F$, rplI-rplT, and rpsB$r p s T)$. Each HEG file was then manually inspected to account for variations in naming conventions. The HEG *. ptt files, which include information regarding the location of the gene within the genome, the gene name, and information about the protein product, are available for download for each strain.

Using code developed in $\mathrm{C}++$, the frequency of each codon within each strain's set of HEG sequences was 
calculated. These frequencies were then used to calculate three metrics representing the codon usage patterns: the relative synonymous codon usage (RSCU), normalized relative synonymous codon usage (NRSCU) and frequency bias (HEG FB) values. The RSCU metric, introduced by Sharp et al. [45], is the observed frequency of the codon divided by the frequency expected under the assumption of equal usage of synonymous codons. The commonly used metric of quantifying codon bias, the CAI value, is derived by referencing the set of RSCU values for a species' HEG sequence set. RSCU values can vary from zero to the number of synonymous codons available for a particular amino acid. The NRSCU value scales the RSCU values such that each codon's value is between zero and one. Thus, each amino acid is weighted equally while still retaining the variance in usage between synonymous codons. The last metric, the HEG FB metric, is the relative frequency of the codon within the set of all codons present in the HEG sequences. The FB values also reflect the variation in synonymous codon usage, but in contrast to the NRSCU metric it also captures biases in amino acid usage within the HEG set. These metrics are calculated as follows:

$$
R S C U_{i j}=\frac{x_{i j}}{\frac{1}{n_{i}} \sum_{j=1}^{n_{i}} x_{i j}} N R S C U_{i j}=\frac{x_{i j}}{\sum_{j=1}^{n_{i}} x_{i j}} H E G \quad F B_{i j}=\frac{x_{i j}}{\sum_{i=1}^{20} \sum_{j=1}^{n_{i}} x_{i j}}
$$

where $x_{i j}$ is the number of occurrences of the $j^{\text {th }}$ codon for the $i^{\text {th }}$ amino acid and $n_{i}$ is the number of synonymous codons which encode for the $i^{\text {th }}$ amino acid. The user can download a table containing the results of these calculations for each strain available through $\mathrm{CBDB}$ as well as a table containing these values for all strains of the selected genus.

The nucleotide and protein sequences of the HEGs for each strain are also available for download from CBDB in FASTA format.

\section{Utility \& discussion}

The motivation behind creating the CBDB site was twofold. Firstly, this site was designed to provide a resource of codon frequency look-up tables for researchers who are investigating translational selection and codon bias in bacteria. Researchers can assess if a nonsynonymous mutation observed is for a codon which is more/less preferred. Secondly, a number of groups, including our own, utilize the phage-host system to explore viral evolution. In particular, $\mathrm{CBDB}$ is a resource for recognizing selection within evolving species.

While CUB-DB [59] includes much of the same data included in CBDB, we wanted to present the data in such a way that one could easily compare the variation in biases between strains and species within a genus.
Secondly, CBDB also contains the FASTA format sequences of the highly expressed genes as well as the gene annotation information, which could then be subsequently analyzed by any of the aforementioned codon analysis software tools. Thus, researchers can go to one repository for this data. In contrast with the resource of the Highly Expressed Genes Database or HEG-DB [61], CBDB includes only the set of HEG identified by Sharp et al. [6] and the calculation of CAI values. Furthermore, this initial release of CBDB contains more species than is available through HEG-DB [61].

Figure 1 shows a screen shot of the CBDB website, showing the results for the first strain listed for the genus Acinetobacter. Each strain's name and NCBI RefSeq number, which includes a hyperlink to the NCBI genome record, is listed followed by links to download: the data table (format "xls), the tab-delimited gene annotation file for the HEG (format *.ptt), the FASTA format amino acid sequences of the HEG set, and the FASTA format nucleotide sequences of the HEG set. Below these links is the data table containing the amino acid abbreviation for each codon and each codon's RSCU, NRSCU, and HEG FB values. At the top of each genus' page is a link to download all of the data tables in a single MS Excel document. Also included is a link to a single MS Excel file which contains the RSCU values for each codon for each strain and an interactive graph to compare biases between strains. The list of all of the available genera appears in the navigation on the left pane of each page and all of the species available for a selected genus. Moreover, the navigation pane includes a link describing the codon bias metrics used and associated references.

While in this release of the database only $300+$ species are available, additional species will be added on a regular basis; all of the annotated bacterial species available through NCBI will be included over the coming months. Furthermore, we are in the process of developing and making publicly available through the site functionality for conducting inter- and intra-species as well as phagehost comparisons of codon usage profiles.

\section{Exploring codon bias with $\mathrm{CBDB}$}

Previous analysis of the variation in codon usage between bacterial species selected just a few representatives of a genus and species [6]. We were interested to see how codon usage varied between species in the same genus as well as between different strains of the same species. For each of the three metrics included in CBDB, we calculated the correlation in codon usage for each pair of strains. A distance matrix was then computed as (1-r)/2 such that the instance of a pair being anticorrelated has a distance of 1 and a perfectly correlated pair has a distance of 0 . The FITCH application of the 


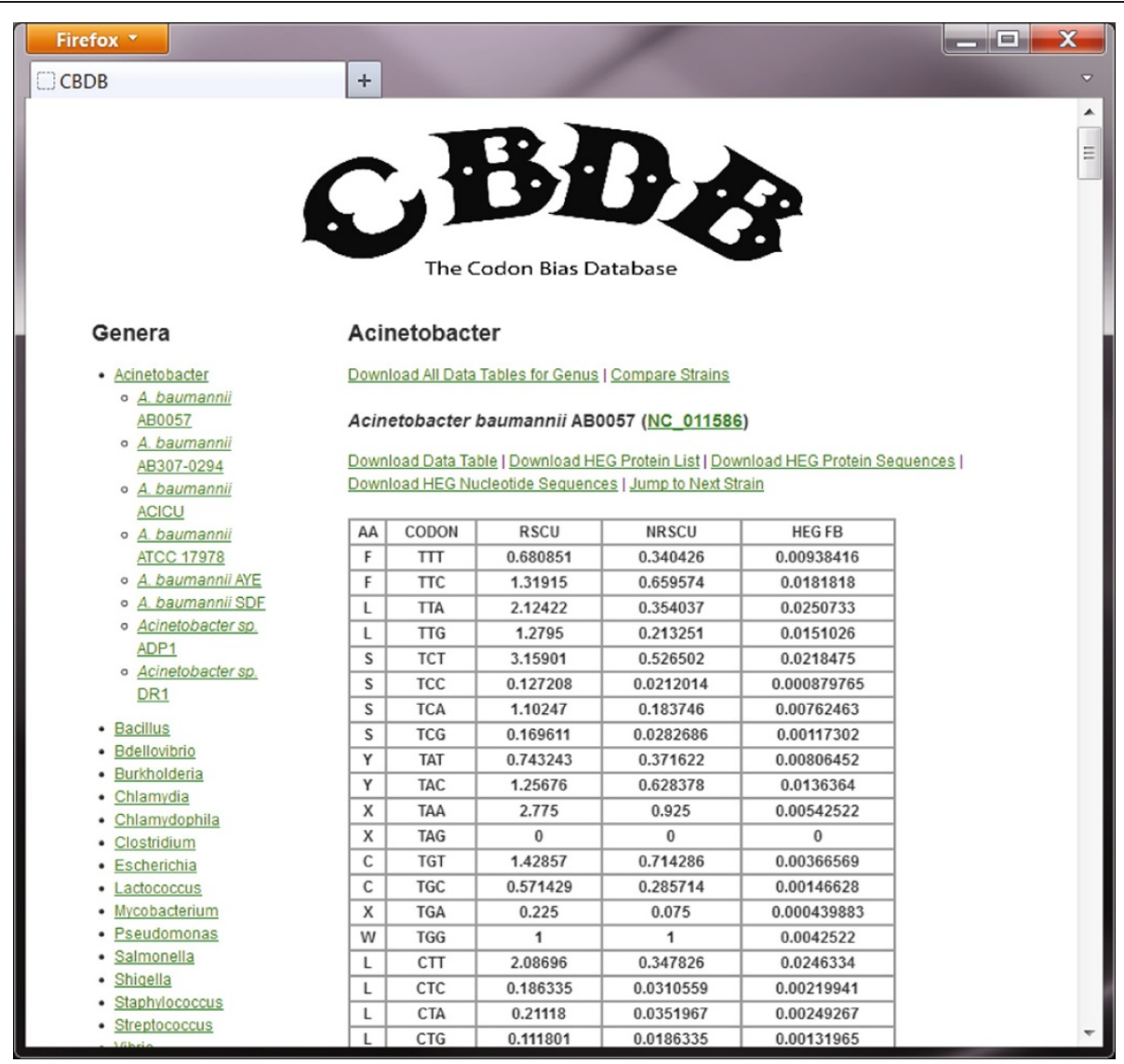

Figure $1 \mathrm{CBDB}$ interface, the results for the first species of the Acinetobacter genus.

PHYLIP package [62] was used to derive a tree to visualize similarities/dissimilarities in codon usage. Figure $2 \mathrm{~A}$ shows the tree derived when the NRSCU values were compared for all strains. (Clades were collapsed when the subtree contained only one genus. Shigella was found to group both with Escherichia and Salmonella. Chlamydia and Chlamydophila were also collapsed into single nodes as some strains were closer to strains of the other species than of their own species.) From Figure 2A, we also identified two branches including Pseudomonas (maroon circle) and Salmonella \& Shigella (orange square) species. The leftmost Salmonella \& Shigella branch, separating the pseudomonads is a single species Salmonella enterica subsp. arizonae serovar 62:z4,z23:str. RSK2980 (GenBank: NC_010067). Also from this visualization of codon usage bias we noticed that the 34 Bacillus strains are interspersed amongst other species in the tree. The two B. lincheniformis and single $B$. clausii strain show a more similar codon usage pattern to the Vibrio fisheri sequences than they do to the other bacilli, as is shown in Figure 2B. Looking at the Bacillus genus data file available from $\mathrm{CBDB}$ which includes statistics for all of the strains, one can see variation in usage between species.

The genera included in this first release vary in the strength of their bias. For instance, as has been previously documented [6], the Chlamydiae phylum does not exhibit a significant bias. The eight Chlamydia and Chlamydophila species examined here do not show a strong bias. ANOVAs were performed for all three statistics revealing that the variation between the species is not statistically significant. The Vibrio species, however, are strongly biased [6] and exhibit differences in codon usage between species. For instance, Figure 3 shows the biases for the 11 Vibrio species examined for the six leucine codons. Referring to the Genomic tRNA database [63], one can find that the non-cholerae species have more TAG-anticodon tRNAs (blue) than any other Leucine tRNAs. This thus can explain the preference within these species for CTA codon. The $V$. cholerae species also have more TAG-anticodon tRNAs than any other Leucine tRNAs, but in contrast to the non-cholerae species they also have CAG-anticodon tRNAs (green) [63]. 


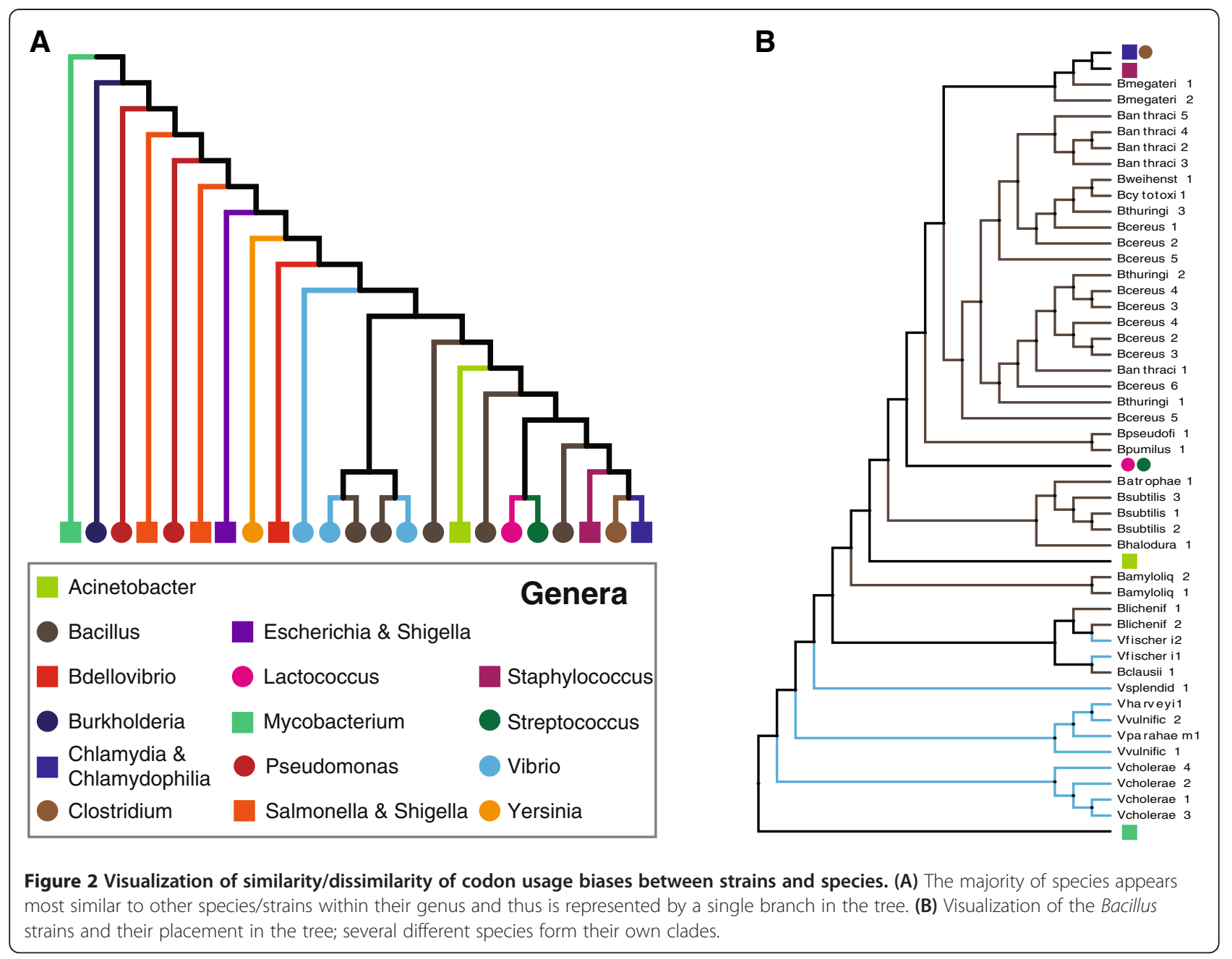

\section{Conclusions}

As recent research has found, the use of particular codons can improve translational accuracy and efficiency in addition to serving as a signal for co-translational protein folding [11,19-34]. Comparing usage between species and strains can expose variation in usage between closely related organisms. Furthermore, this site serves as a resource for studying the mechanisms shaping codon usage within bacteriophages. The Codon Bias Database (CBDB) provides a centralized repository of

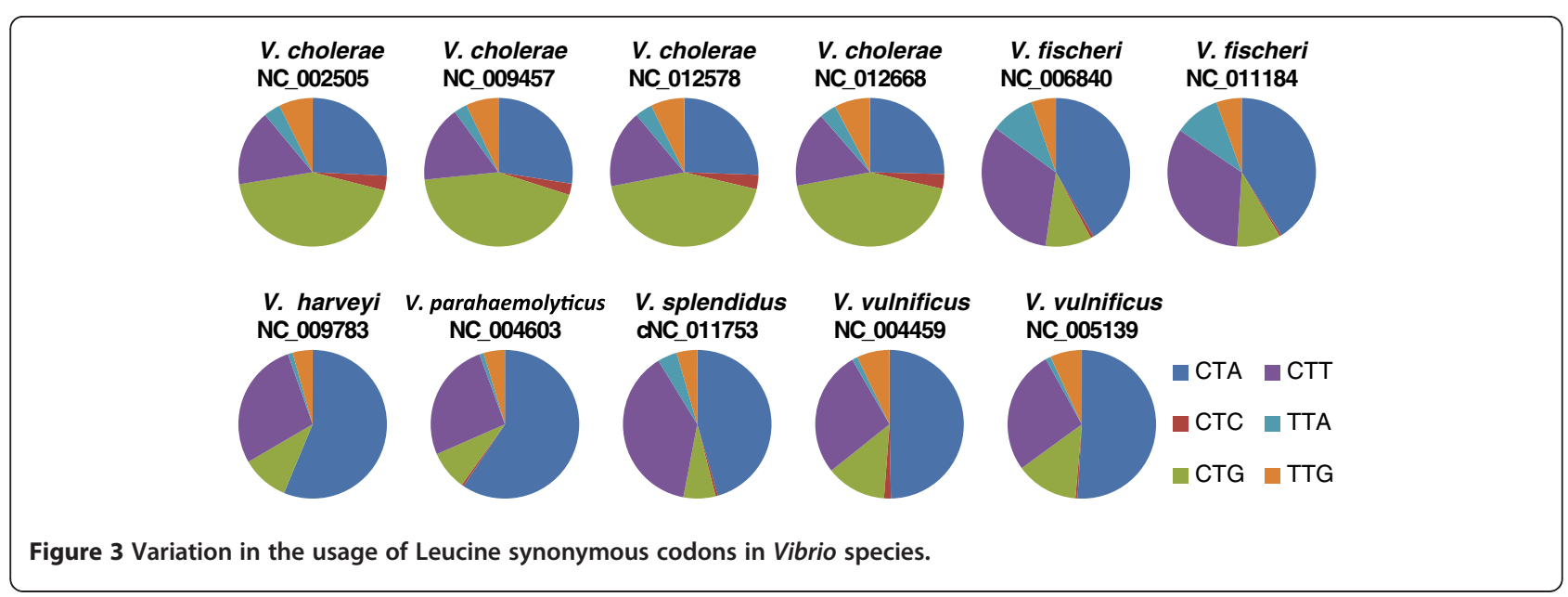


look-up tables and codon usage bias measures for a wide variety of genera, species and strains, facilitating comparisons in codon usage between closely related species such as the one presented here.

\section{Availability and requirements}

The Codon Bias Database (CBDB) is freely available without restriction at www.cbdb.info. This website has been tested with browsers of Safari, Internet Explorer and Firefox.

\section{Competing interests}

The authors declare that they have no competing interests.

\section{Authors contributions}

$\mathrm{AH}$ and JS collected the data as well as participated in the design of the site and writing of the manuscript. CP conceived of the study and site and developed the code used for generating data and analyses in addition to participating in the design of the site and writing of the manuscript. All authors read and approved the final manuscript.

\section{Acknowledgments}

This study was partially funded by a research support grant from Loyola University Chicago (CP).

\section{Author details}

'Department of Biology, Loyola University Chicago, 1032 W Sheridan Road, Chicago IL 60660, USA. Bioinformatics Program, Loyola University Chicago, 1032 W Sheridan Road, Chicago IL 60660, USA. ${ }^{3}$ Department of Computer Science, Loyola University Chicago, 820 N Michigan Avenue, Chicago IL 60611, USA.

Received: 9 January 2012 Accepted: 26 March 2012 Published: 26 April 2012

\section{References}

1. Sueoka N: On the genetic basis of variation and heterogeneity of DNA base composition. Proc Natl Acad Sci USA 1962, 34:95-114.

2. Bennetzen JL, Hall BD: Codon selection in yeast. J Biol Chem 1982, 257:3026-3031.

3. Gautier C: Compositional bias in DNA. Curr Opin Genet Dev 2000 10:656-661.

4. dos Reis M, Savva R, Wernisch L: Solving the riddle of codon usage preferences: a test for translational selection. Nucleic Acids Res 2004, 32:5036-5044.

5. Urrutia AO, Hurst LD: Codon usage bias covaries with expression breadth and the rate of synonymous evolution in humans, but this is not evidence for selection. Genetics 2001, 159:1191-1199.

6. Sharp PM, Bailes E, Grocock RJ, Peden JF, Sockett RE: Variation in the strength of selected codon usage bias among bacteria. Nucleic Acids Res 2005, 33:1141-1153.

7. Bulmer M: The selection-mutation-drift theory of synonymous codon usage. Genetics 1991, 129:897-907.

8. Palidwor GA, Perkins TJ, Xia X: A general model of codon bias due to GC mutational bias. PLoS One 2010, 5:e13431.

9. Supek F, Škunca N, Repar J, Vlahoviček K, Šmuc T: Translational selection is ubiquitous in prokaryotes. PLoS Genet 2010, 6:e1001004.

10. Hershberg R, Petrov DA: Selection on codon bias. Annu Rev Genet 2008, 42:287-299.

11. Shah $P$, Gilchrist MA: Explaining complex codon usage patterns with selection for translational efficiency, mutation bias, and genetic drift. Proc Natl Acad Sci U S A 2011, 108:10231-10236.

12. Bulmer M: Coevolution of codon usage and transfer RNA abundance. Nature 1987, 325:728-730.

13. Ikemura T: Correlation between the abundance of transfer RNAs and the occurrence of the respective codons in its protein genes: A proposal for a synonymous codon choice that is optimal for translational system. $J$ Mol Biol 1981, 151:389-409.
14. Ikemura T: Codon usage and tRNA content in unicellular and multicellular organisms. Mol Biol Evol 1985, 2:13-34.

15. Kanaya S, Yamada Y, Kudo Y, Ikemura T: Studies of codon usage and tRNA genes of 18 unicellular organisms and quantification of Bacillus subtilis tRNAs: gene expression level and species-specific diversity of codon usage based on multivariate analysis. Gene 1999, 238:143-155.

16. Burgess-Brown NA, Sharma S, Sobott F, Loenarz C, Oppermann U, Gileadi O: Codon optimaization can improve expression of human genes in Escherichia coli: A multi-gene study. Protein Expr Purif 2008, 59:94-102.

17. Maertens B, Spriestersbach A, von Groll U, Roth U, Kubicek J, Gerrits M, Graf M, Liss M, Daubert D, Wagner R, Schäfer F: Gene optimization mechanisms: a multi-gene study reveals a high success rate of full-length human proteins expressed in Escherichia coli. Protein Sci 2010, 19:1312-1326.

18. Welch M, Govindarajan S, Ness JE, Villalobos A, Gurney A, Minshull J, Gustafsson C: Design parameters to control synthetic gene expression in Escherichia coli. PLoS One 2009, 4:e7002.

19. Akashi H: Synonymous codon usage in Drosophila melanogaster: natural selection and translational accuracy. Genetics 1994, 136:927-935.

20. Marais G, Duret L: Synonymous codon usage, accuracy of translation, and gene length in Caenorhabditis elegans. J Mol Evol 2001, 52:275-280.

21. Stoletzki N, Eyre-Walker A: Synonymous codon usage in Escherichia coli: selection for translational accuracy. Mol Biol Evol 2007, 24:374-381.

22. Zhou T, Weems M, Wilke CO: Translationally optimal codons associate with structurally sensitive sites in proteins. Mol Biol Evol 2009, 26:1571-1580.

23. Sharp PM, Li W-H: The codon adaptation index: a measure of directional synonymous codon usage, and its potential applications. Nucleic Acids Res 1987, 15:1281-1295.

24. dos Reis M, Wernisch L, Savva R: Unexpected correlations between gene expression and codon usage bias from microarray data for the whole Escherichia coli K-12 genome. Nucleic Acids Res 2003, 22:2437-2446.

25. Brockmann R, Beyer A, Heinisch JJ, Wilhelm T: Posttranscriptional expression regulation: what determines translation rates? PLoS Comput Biol 2007, 3:e57.

26. Tuller T, Carmi A, Vestsigian K, Navon S, Dorfan Y, Zaborske J, Pan T, Dahan $\mathrm{O}$, Furman I, Pilpel Y: An evolutionary conserved mechanism for controlling the efficiency of protein translation. Cell 2010, 141:344-354.

27. Zhang Z, Zhou L, Hu L, Zhu Y, Xu H, Liu Y, Chen X, Yi X, Kong X, Hurst LD: Nonsense-mediated decay targets have multiple sequence-related features that can inhibit translation. Mol Syst Biol 2010, 6:442.

28. Gingold H, Pilpel Y: Determinants of translation efficiency and accuracy. Mol Syst Biol 2011, 7:481.

29. Plotkin JB, Kudla G: Synonymous but not the same: the causes and consequences of codon bias. Nat Rev Genet 2011, 12:32-42.

30. Kudla G, Murray AW, Tollervey D, Plotkin JB: Coding-sequence determinants of gene expression in Escherichia coli. Science 2009, 324:255-258.

31. Gilchrist MA, Shah P, Zaretzki R: Measuring and detecting molecular adaptation in codon usage against nonsense errors during protein translation. Genetics 2009, 183:1493-1505.

32. Tsai CJ, Sauna ZE, Kimchi-Sarfaty C, Ambudkar SV, Gottesman MM, Nussinov $\mathrm{R}$ : Synonymous mutations and ribosome stalling can lead to altered folding pathways and distinct minima. J Mol Biol 2008, 383:281-291.

33. Marin M: Folding at the rhythm of the rare codon beat. Biotechnol J 2008, 3:1047-1057.

34. Ivanov IG, Saraffova AA, Abouhaidar MG: Unusual effect of clusters of rare arginine (AGG) codons on the expression of human interferon alpha 1 gene in Escherichia coli. Int J Biochem Cell Biol 1997, 29:659-666.

35. Karlin S, Doerfler W, Cardon LR: Why is CpG suppressed in the genomes of virtually all small eukaryotic viruses but not in those of large eukaryotic viruses? J Virol 1994, 68:2889-2897.

36. Shackelton LA, Parrish CR, Holmes EC: Evolutionary basis of codon usage and nucleotide composition bias in vertebrate DNA viruses. $J \mathrm{Mol}$ Evol 2006, 62:551-563.

37. Greenbaum BD, Levine AJ, Bhanot G, Rabadan R: Patterns of evolution and host gene mimicry in influenza and other RNA viruses. PLoS Pathog 2008, 4:e1000079.

38. Schubert AM, Putonti C: Evolution of the sequence composition of Flaviviruses. Infect Genet Evol 2010, 10:129-136.

39. Jenkins GM, Holmes EC: The extent of codon usage bias in human RNA viruses and its evolutionary origin. Vir Res 2003, 92:1-7. 
40. Kunisawa T, Kanaya S, Kutter E: Comparison of synonymous codon distribution patterns of bacteriophage and host genomes. DNA Res 1998, 5:319-326.

41. Lucks JB, Nelson DR, Kudla GR, Plotkin JB: Genome landscapes and bacteriophage codon usage. PLoS Comput Biol 2008, 4:e1000001.

42. Carbone $\mathrm{A}$ : Codon bias is a major factor explaining phage evolution in translationally biased hosts. J Mol Evol 2008, 66:210-223.

43. McLachlan AD, Staden R, Boswell DR: A method for measuring the nonrandom bias of a codon usage table. Nucleic Acids Res 1984, 12:9567-9575.

44. Clarke TF IV, Clark PL: Rare codons cluster. PLoS One 2008, 3:e3412.

45. Sharp PM, Tuohy TMF, Mosurski KR: Codon usage in yeast: Cluster analysis clearly differentiates highly and lowly expressed genes. Nucleic Acids Res 1986, 14:5125-5143.

46. Carbone A, Zinovyev A, Képès F: Codon Adaptation Index as a measure of dominating codon bias. Bioinformatics 2003, 19:2005-2015.

47. Lee $S$, Weon S, Lee S, Kang C: Relative codon adaptation index, a sensitive measure of codon usage bias. Evol Bioinform Online 2010, 6:47-55.

48. Sharp PM, Emery LR, Zeng K: Forces that influence the evolution of codon bias. Phil Trans R Soc B 2010, 365:1203-1212.

49. Puigbò $P$, Bravo IG, Garcia-Vallvé S: CAlcal: a combined set of tools to access codon usage adaptation. Biol Direct 2008, 3:38.

50. Harnady M, Wilson SA, Zaneveld J, Sueoka N, Knight R: CodonExplorer: an online tool for analyzing codon usage and sequence composition, scaling from genes to genomes. Bioinformatics 2009, 25:1331-1332.

51. Peden J: CodonW [http://codonw.sourceforge.net/]

52. Puigbò $P$, Bravo IG, Garcia-Vallvé S: E-CAl: a novel server to estimate an expected value of Codon Adaptation Index (eCAl). BMC Bioinforma 2008, 9:65.

53. Angellotti MC, Bhuiyan SB, Chen G, Wan XF: CodonO: codon usage bias analysis within and across genomes. Nucleic Acids Res 2007, 35:W132-W136.

54. Grote A, Hiller K, Scheer M, Münch R, Nörtemann B, Hempel DC, Jahn D: JCat: a novel tool to adapt codon usage of a target gene to its potential expression host. Nucleic Acids Res 2005, 33:W526-W531.

55. Fuhrmann M, Hausherr A, Ferbitz L, Schödl T, Heitzer M, Hegemann P: Monitoring dynamic expression of nuclear genes in Chlamydomonas reinhardtii by using a synthetic luciferase reporter gene. Plant Mol Biol 2004, 55:869-881.

56. Nakamura $Y$, Gojobori T, Ikemura T: Codon usage tabulated from international DNA sequence databases: status for the year 2000. Nucleic Acids Res 2000, 28:292.

57. MGCUD: Microbial Genome Codon Usage Database http://bioinformatics. forsyth.org/mgcud/

58. Zhu W, Lomsadze A, Borodovsky M: Ab initio gene identification in metagenomic sequences. Nucleic Acids Res 2010, 38:e132.

59. Raiford D: CUB-DB: Codon Usage Database http://web-dev.cs.umt.edu/ cub_db/

60. NCBI FTP ftp://ftp.ncbi.nlm.nih.gov/

61. Puigbò P, Romeu A, Garcia-Vallvé S: HEG-DB: a database of predicted highly expressed genes in prokaryotic complete genomes under translational selection. Nucleic Acids Res 2008, 36:D524-D527.

62. Felsenstein J: PHYLIP http://evolution.genetics.washington.edu/phylip.html

63. Chan PP, Lowe TM: GtRNAdb: A database of transfer RNA genes detected in genomic sequence. Nucleic Acids Res 2009, 37:D93-D97.

\section{Submit your next manuscript to BioMed Central and take full advantage of:}

- Convenient online submission

- Thorough peer review

- No space constraints or color figure charges

- Immediate publication on acceptance

- Inclusion in PubMed, CAS, Scopus and Google Scholar

- Research which is freely available for redistribution

Submit your manuscript at www.biomedcentral.com/submit 